\title{
Phase transitions and critical behaviour in one-dimensional nonequilibrium kinetic Ising models with branching annihilating random walk of kinks.
}

\author{
Nora Menyhárd \\ Research Institute for Solid State Physics, H-1525 Budapest,P.O.Box 49, Hungary \\ Géza Ódor \\ Research Institute for Materials Science, H-1525 Budapest, P.O.Box 49, Hungary
}

\begin{abstract}
One-dimensional non-equilibrium kinetic Ising models evolving under the competing effect of spin flips at zero temperature and nearest neighbour spin exchanges exhibiting a parity-conserving (PC) phase transition on the level of kinks are now further investigated, numerically, from the point of view of the underlying spin system. Critical exponents characterising its statics and dynamics are reported. It is found that the influence of the $\mathrm{PC}$ transition on the critical exponents of the spins is strong and the origin of drastic changes as compared to the Glauber-Ising case can be traced back to the hyperscaling law stemming from directed percolation. Effect of an external magnetic field, leading to directed percolation type behaviour on the level of kinks, is also studied, mainly via the generalized mean field approximation.
\end{abstract}

05.70.Ln, $05.50 .+\mathrm{q}$

\section{INTRODUCTION}

Nonequilibrium phase transitions have attracted great interest lately. A variety of sytems studied seem to belong to the universality class of directed percolation (DP) [1 the DP universality class is very robust, among others the transitions in branching annihilating random walk (BARW) models with an odd number of offsprings in the process $A \rightarrow A+n A$ belong to it [5]. Numerical studies by Grassberger et al. [6,7] of probabilistic cellular automata models in one dimension involving the processes $k \rightarrow 3 k$ and $2 k \rightarrow 0$ ( $k$ stands for kink) have revealed, however, a new universality class of dynamic phase transitions. Both time-dependent and steady-state simulations have resulted non-DP values for the relevant critical exponents. This so called parity-conserving (PC) phase transition has been found since in a variety of models. The $n=4$ BARW model has been studied in the greatest detail and accuracy by Jensen [8]. In a previous paper [9] one of the the authors has introduced a family of nonequilibrium kinetic Ising models (NEKIM) showing the same phenomenon, while quite recently, with an appropriate modification of the original BARW model (suggested already in ref. [9]), the $n=2$ BARW model has been shown to exhibit the PC transition as well [10]. The two-component interacting monomer-dimer model introduced by Kim and Park [11 [13] represents a more complex sytem with a PC-type phase transition.

Formerly, combinations of Glauber [14] and Kawasaki [15] kinetics were introduced with the aim of investigating temperature-driven nonequilibrium phase transitions [16,17]. In ref. [9], however, spin-flip kinetics was taken at $T=0$ alternatingly with random nearest-neighbour spin-exchanges ( Kawasaki-exchange at $T=\infty$ ). The mean-field limit of this model together with results of the generalised mean-field theory (GMF) have been presented in [18].

In the present paper we further investigate NEKIM but now from the point of view of the underlying 1D spin system with the aim of getting some more insight into the nature of the PC transition. Results of computer simulations are presented for different critical exponents connected with the structure factor. The new computational technique based on short-time dynamics and finite size scaling, as introduced and applied by Li,Schülke and Zheng [19,20] for calculating moments of the magnetisation and the time dependent Binder cumulant, is also applied here besides the usual finite size scaling (FSS) and time dependent simulations. In all our numerical studies the initial state is random with zero magnetisation and concerning finite size effects the use of antiperiodic boundary conditions instead of the usual periodic ones will prove to be essential.

In the field of domain-growth kinetics it has long been accepted that the scaling exponent of $L(t)$, the characteristic domain size, is equal to $1 / 2$ if the order parameter is non-conserving. Now let us restrict ourselves to a $1 \mathrm{D}$ Ising spin chain of length $L$ and define the structure factor as usual : $S(0, t)=L\left[<M^{2}>-<M>^{2}\right], M=\frac{1}{L} \sum_{i} s_{i},\left(s_{i}= \pm 1\right)$. If the conditions of validity of scaling are fulfilled [21] then

$$
S(0, t)) \propto[L(t)]^{d} ; \quad L(t) \propto t^{x}
$$

where $d=1$ now and $x=1 / 2$. An other usually considered quantity is the excess energy of a monodomain sample at the temperature of quench, which in our case is proportional to the kink density $\left.n(t)=\frac{1}{L}<\sum_{i} \frac{1}{2}\left(1-s_{1} s_{i+1}\right)\right\rangle$. 


$$
n(t) \propto \frac{1}{L(t)} \propto t^{-y}
$$

with $y=1 / 2$ in the Glauber-Ising case, expressing the well-known dependence on time of annihilating random walk.

It is also well-known, that the parameter $p_{T}=e^{-4 J / k T}$ can be regarded as the quantity measuring the deviation from the critical temperature $T=0$ of the 1D Ising model, and the usual (equilibrium) critical exponents can be defined as powers of $p_{T}$. From exact solution the critical exponents of the spin- susceptibility, coherence length and magnetisation are known to be $\gamma=\nu=1 / 2, \beta=0$, respectively. Fisher's static scaling law $\gamma=d \nu-2 \beta$ is valid. Moreover, the dynamic critical exponent $Z$ is equal to 2 .

Instead of Glauber kinetics, let us apply now the nonequilibrium NEKIM kinetics, and investigate the Ising system at and in the immediate neighbourhood of one of its PC points. On the level of kinks the PC point, separating the active and absorbing phases, is a second order transition point, while from the point of view of spins the absorbing phase consists of a multitude of 1D Ising critical points, which ends at the PC point. We are interested in the behaviour of the spin system at this end-point.

The result is the following: the critical kink-dynamics has strong influence on the spin-kinetics and even on its statics. Domain growth is governed by criticality: $\mathrm{x}=1 / \mathrm{Z}$, but the dynamic exponent $Z$ changes from $Z=2$ to $Z=1.75$. As to statics, $\gamma=\nu=.444$ while $\beta=0$ and Fisher's scaling law remain valid.

More spectacular is the change of the above $y$-exponent: $y=x / 2 ; x=.57$ and similarly the exponent $\alpha_{n}$ of the finite-temperature kink-density: $\lim _{t \rightarrow \infty} n\left(t, p_{T}\right) \propto p_{T}{ }^{\alpha_{n}}$, which is $1 / 2$ in the Glauber-Ising limit, decreases to $\alpha_{n}=\gamma / 2=.222$. We shall argue that this factor of 2 between magnetic- and kink-exponents has its origin in the hyperscaling law, introduced for DP by Grassberger and de la Torre [1], which connects exponents of time-dependent kink density and cluster-size.

These numerical results will be reported in detail in the following. Moreover, we shall present further results, mainly from the side of applying GMF approximations, by introducing a magnetic field-term into the spin-flip probability, which causes the PC transition to become DP-like [13].

\section{THE MODEL}

The model we will investigate here is a one-dimensional kinetic Ising model evolving by a combined spin-flip and spin-exchange dynamics as described in [9]. The spin-flip transition rate in one-dimension for spin $s_{i}$ sitting at site $i$ is:

$$
w_{i}=\frac{\Gamma}{2}\left(1+\delta s_{i-1} s_{i+1}\right)\left(1-\frac{\gamma}{2} s_{i}\left(s_{i-1}+s_{i+1}\right)\right)
$$

where $\gamma=\tanh 2 J / k T$ ( $J$ denoting the coupling constant in the Ising Hamiltonian), $\Gamma$ and $\delta$ are further parameters. While in [9] $T=0(\gamma=1)$ has been taken, we shall consider now finite temperature effects, too. Instead of $\gamma$ the parameter $p_{T}$ will be used in the following. The three independent rates:

$$
w_{\text {indif }}=\frac{\Gamma}{2}(1-\delta), w_{\text {oppo }}=\Gamma(1+\delta) \frac{1}{1+p_{T}}, w_{\text {same }}=\Gamma(1+\delta) \frac{p_{T}}{1+p_{T}},
$$

where the subscripts of $w$ refer to the three possible neighbourhoods of a given spin, are responsible - on the level of domain walls - for random walk, annihilation and pairwise creation (inside of a domain) of kinks, respectively. The other ingredient of NEKIM has been a spin-exchange transition rate of neighbouring spins ( the Kawasaki 15 rate at $T=\infty)$ :

$$
w_{i i+1}=\frac{1}{2} p_{e x}\left[1-s_{i} s_{i+1}\right]
$$

where $p_{e x}$ is the probability of spin exchange.

Spin-flip and spin-exchange have been applied alternatingly at each time step, the spin-flip part has been applied using two-sublattice updating, while making $L \mathrm{MC}$ attempts at random ( $L$ denotes the size of the chain) has been counted as one time-step of exchange updating. In this system, at $T=0, \mathrm{PC}$ type phase transition takes place. In 9 we have started from a random initial state and spotted the phase boundary in the $(\delta$, pex $)$ plane. In the following we will choose a typical point on this phase diagram and make simulations at and around this point, fixing $\Gamma$ and $p_{e x}$ and changing only $\delta$. The parameters chosen are: $\Gamma=.35$, pex $=.3, \delta_{c}=-.395(2)$. We note here that $\Gamma$ appeared as $1 / \Gamma$ in ref. [9] and $\delta_{c}$ has a more accurate value now than previously. 


\section{SCALING FORMS AND LAWS}

In the following we will be interested in two quantities characterising the behaviour of the NEKIM, namely the structure factor or spin-susceptibility and the kink-density under the conditions of a quench. Thus, in contrast to the usually considered evolution from a pair of kinks, we will restrict ourselves to completely random initial states ( $T=\infty, M(0)=0$ ) and follow the development of the system via the rules described in the previous section. With $p_{T}$ defined above and $\epsilon=\left|\delta-\delta_{c}\right|$, where $\delta<0$ is the parameter which drives the phase transition in the present case, the scaling forms for $S$ and $n$ are as follows:

$$
\begin{aligned}
& S\left(p_{T}, \epsilon, L, t\right)=t^{x} f\left(\frac{t^{\frac{1}{z_{c}}}}{\xi}, \frac{t^{\frac{1}{z}}}{\xi_{\perp}}, \frac{\xi}{L}, \frac{\xi_{\perp}}{L}\right) \\
& n\left(p_{T}, \epsilon, L, t\right)=t^{-y} g\left(\frac{t^{\frac{1}{z_{c}}}}{\xi}, \frac{t^{\frac{1}{Z}}}{\xi_{\perp}}, \frac{\xi}{L}, \frac{\xi_{\perp}}{L}\right)
\end{aligned}
$$

with $\xi=p_{T}^{-\nu}, \xi_{\perp}=\epsilon^{-\nu_{\perp}}$. The exponents connected with $\epsilon$ have been written using the notation of directed percolation, while those related to the temperature factor $p_{T}$ are written in the notation of equilibrium Ising system. $Z$ and $Z_{c}$ are the respective dynamic critical exponents and we allow for the possibility that they differ (though this will turn out not to be the case).

\section{A. The large L case}

Let us first take the limit $L \rightarrow \infty$. Then the dependences on $\xi / L$ and $\xi_{\perp} / L$ can be neglected in eqs (6),(何). If furthermore $\xi \rightarrow \infty, \xi_{\perp} \rightarrow \infty$ the forms valid at the critical point $\epsilon=0, p_{T}=0$ are obtained: $S_{c}(t) \propto t^{x}$ and $n_{c}(t) \propto t^{-y}$. Fig.1. shows $S_{c}(t) \propto t^{x}$, with the result $x=.570(1)$. The same statistics has led now for the exponent of the kink density to the value $y=.285(1)$ (denoted $\alpha$ in [9]).

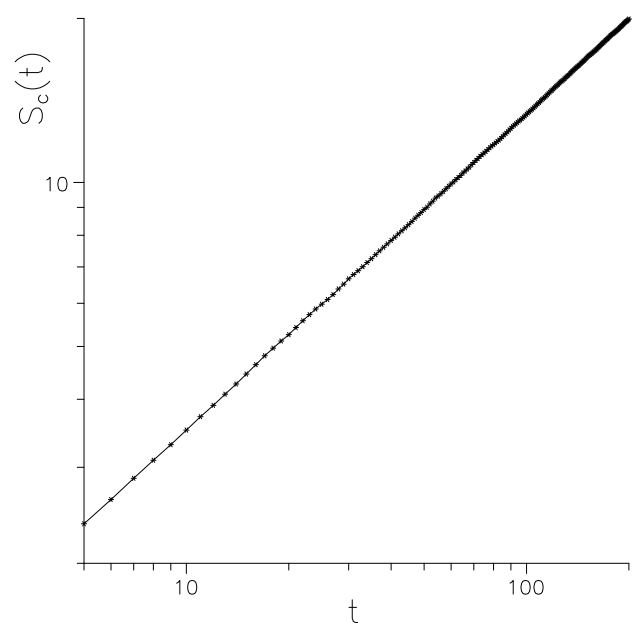

FIG. 1. The sructure factor as a function of time at a typical phase transition point of the NEKIM phase diagram. $L=128$ and the number of independent initial configurations with zero magnetisation was $10^{5}$. Here $t_{\max }=200$ and finite size effects start to set in at about $t=1000$. The straight line is fit with $x=.570$. 
We have also made simulations for the same quantities with $L=8000$ up to $t=3 \times 10^{4}$ and averaging over 2000 initial states. with similar result but with higher error and more than 100 times bigger computer time.It is worth noticing, that within error $x=2 y$.

Keeping $p_{T}=0$, near $\delta_{c}$ in the active phase eq.(7) gives, for $t \rightarrow \infty$ the order parameter $n(\epsilon) \propto \epsilon^{\beta_{n}}$ with the scaling law [7]

$$
\beta_{n}=\nu_{\perp} Z y \text {. }
$$

A similar scaling relation can be obtained from (6), which yields for $t \rightarrow \infty: S(\epsilon) \propto \epsilon^{-\Theta}$ with

$$
\Theta=\nu_{\perp} Z x \text {. }
$$

The divergence of the spin-susceptibility as a function of $\epsilon$ is understandable: in the subcritical regime (i.e. for $\left.|\delta|<\left|\delta_{c}\right|\right)$ it is infinite $(T \rightarrow 0, t \rightarrow \infty)$, because the whole subcritical region is a plane of $1 d$ critical (Ising) points. In order to get $\Theta$ directly, we have made simulations for $S(\epsilon, t)$ around the chosen PC point in the interval $\epsilon=.02-.13$ as shown on Fig.2.

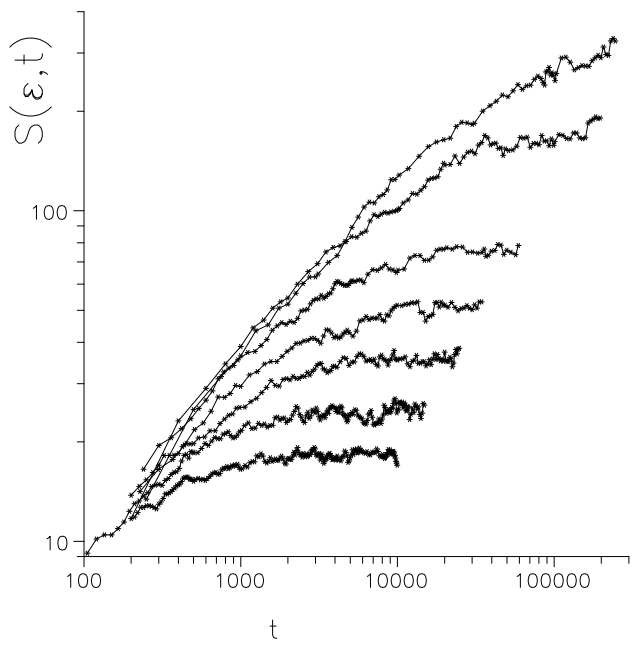

FIG. 2. The structure factor $S(\epsilon, t)$ in the active phase in the vicinity of the PC transition point for the following values of $\epsilon: .13, .11, .09, .075, .06, .04, .03$ (from bottom to top). L was varied between 2000 and 8000 to avoid finite size effects before saturation sets in. Number of independent states in averaging: typically 1000.

The (time-averaged) saturation values of $S$ yield the exponent $\Theta=1.9(1)$. From the same runs as above we have obtained now $\beta_{n}=.88(4)$. Thus within error $\Theta=2 \beta_{n}$, which relation also follows from eqs. (8) and (9) with $x=2 y$. It is worth noting here that on the basis of the divergence of the spin susceptibility, $S(\epsilon)$, the PC transition point (as endpoint of a line of first order transitions) can be found, without any reference to kinks.

Taking now $\epsilon=0$ and keeping $p_{T}$ finite in the limit $t \rightarrow \infty$ we get from eq. (7) $n\left(p_{T}\right) \propto p_{T}^{\alpha_{n}}$ and the scaling relation

$$
\alpha_{n}=y \nu Z_{c}
$$

Fig. 3 shows $n\left(t, p_{T}\right)$ as a function of $t$ at different values of $p_{T}$ at $\epsilon=0$; the level-off values could be fitted with $\alpha_{n}=.222(5)$. This result is in accord with the value for the $n=4$ BARW reported by Jensen $[8]\left(1 / \delta_{h}\right.$ in his notation).

Similarly, taking eq.(6) at $\epsilon=0$ and in the limit $t \rightarrow \infty$ the spin-susceptibility arises: $\chi \propto p_{T}^{-\gamma}$ together with the scaling relation

$$
\gamma=x \nu Z_{c}
$$


On Fig.4 $S\left(p_{T}, \epsilon=0, t\right)$ is plotted for different values of $p_{T}$.

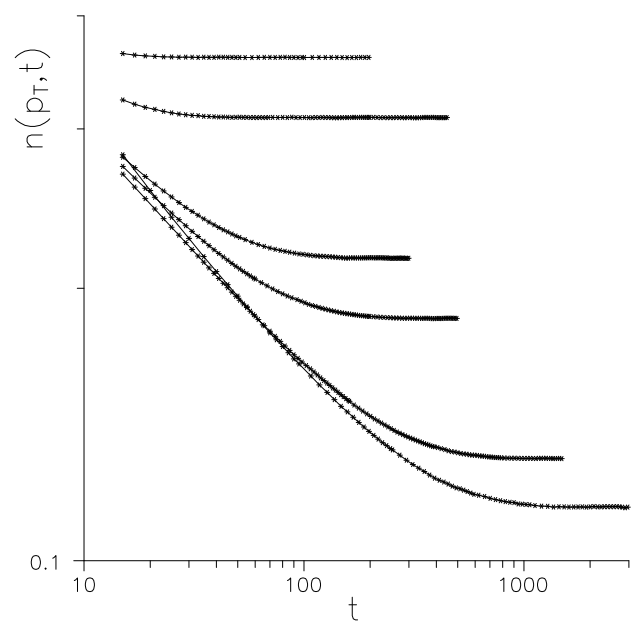

FIG. 3. The kink-density as a function of temperature and time at the PC point.The curves are for $p_{T}=10^{-1}, 5.0 \times 10^{-2}, 10^{-2}, 5.0 \times 10^{-3}, 10^{-3}, 5.0 \times 10^{-4}$ from top to bottom. $L=2000$ and the number of samples in averaging typically $2 \times 10^{4}$.

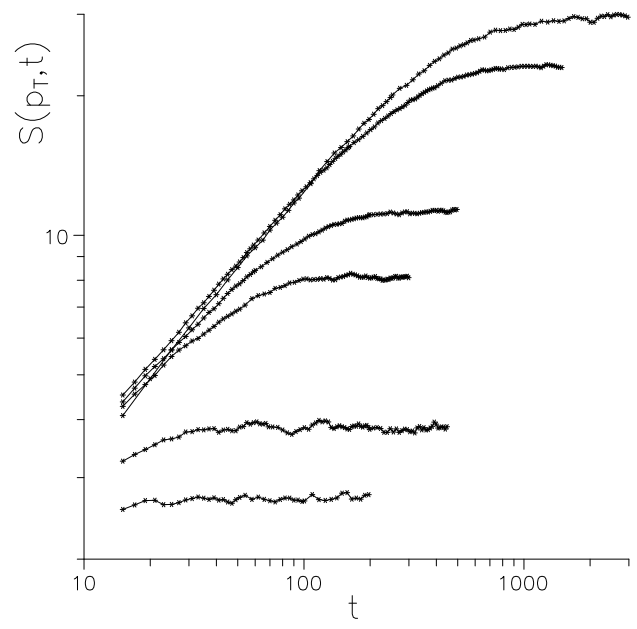

FIG. 4. The structure factor $S\left(p_{T}, t\right)$ for different values of $p_{T}$. Details of simulation as of Fig. 3 except that $p_{T}$ is decreasing from bottom to top here.

The temperature-dependent simulations have been performed in the range $p_{T}=1.10^{-2}-5.10^{-5}$. The level-off values seen on Fig.5, where the data for the two highest temperatures $\left(p_{T}=1.10^{-1}, 5.10^{-2}\right)$ were discarded , yield the exponent of the spin-susceptibility at the PC point as $\gamma=.445(5)$. This value is in accord, within error, with the scaling laws (10) and (11) which predict, using the relation $x=2 y$ found above, $\gamma=2 \alpha_{n}=.444$. 


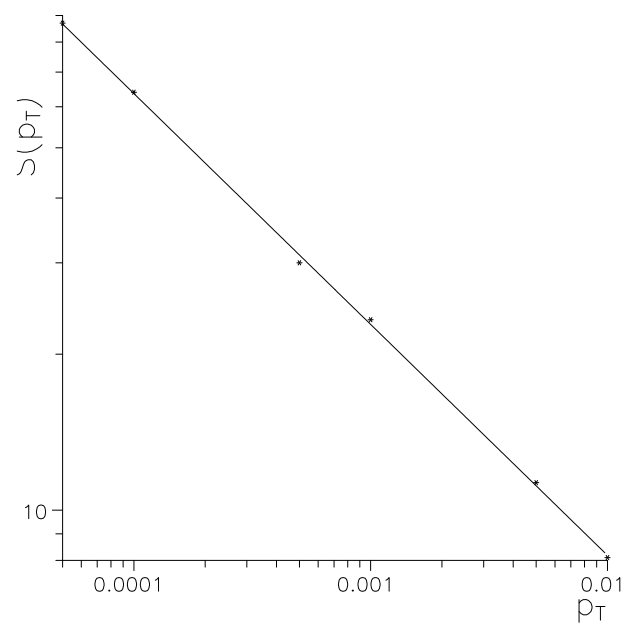

FIG. 5. The saturation values of Fig. 4 time-averaged, on a double-logarithmic scale. Data for $p_{T}=10^{-4}, 5 \times 10^{-5}$, not shown on Fig.4, have been included. Straight line: fit of data points with $\gamma=.445$

It is to be noted, that due to the non-self-averaging property of the structure factor [22], all exponents connected with this quantity have much larger statistical errors than the ones connected with the kink density, and the same applies to the time-dependent simulations. This explains the large fluctuations exhibited on Fig.2 and Fig.4 in comparison with e.g. Fig.3.

\section{B. Static finite size scaling}

Finite size scaling will be used to find numerical values for some more exponents. The static FSS limit requires to take first the limit $t \rightarrow \infty$ and to suppose $L \ll \xi, \xi_{\perp}$. Let us consider now the two possible orders of limits to reach the PC point: a). $p_{T}=0, \epsilon \rightarrow 0$ and b). $\epsilon=0, p_{T} \rightarrow 0$.

Case a).

Eqs (6) and (7) lead to the expressions: $\lim _{\epsilon \rightarrow 0} S(\epsilon, L)=L^{\frac{\Theta}{\nu_{\perp}}} f^{\prime \prime}\left(\frac{L}{\xi_{\perp}}\right) \propto L^{\frac{\Theta}{\nu_{\perp}}}$ and $\lim _{\epsilon \rightarrow 0} n(\epsilon, L)=L^{\frac{-\beta_{n}}{\nu_{\perp}}} g^{\prime \prime}\left(\frac{L}{\xi_{\perp}}\right) \propto L^{\frac{-\beta_{n}}{\nu_{\perp}}}$, respectively.

Case b).

From eqs (6) and (7) we get now $\lim _{p_{T} \rightarrow 0} S\left(p_{T}, L\right)=L^{\frac{\gamma}{\nu}} \tilde{f}^{\prime \prime}\left(\frac{L}{\xi}\right) \propto L^{\frac{\gamma}{\nu}}$ and $\lim _{p_{T} \rightarrow 0} n\left(p_{T}, L\right)=L^{-\frac{\alpha_{n}}{\nu}} \tilde{g}^{\prime \prime}\left(\frac{L}{\xi}\right) \propto L^{-\frac{\alpha_{n}}{\nu}}$, respectively.

Within error we have not found any (numerical) evidence against the supposition that the order of limits $\epsilon \rightarrow 0$ and $p_{T} \rightarrow 0$ were interchangeable, the same fixed point seems to be reached in both cases. (Actually this fact has expressed itself already in our finding, according to which the time-exponents $x$ and $y$ are also the same independently from where we approach the limit $\epsilon=0, p_{T}=0$; the difference should have been seen in the preasymptotic timedependence. E.g. for very small values of $p_{T}$ at $\epsilon=0 x=.57, y=.28$ was always clearly seen in the early-time behaviour of $S(t)$ and $n(t)$, resp.).

Thus the above relations lead to the following scaling equalities:

$$
\frac{\gamma}{\nu}=\frac{\Theta}{\nu_{\perp}}, \quad \frac{\beta_{n}}{\nu_{\perp}}=\frac{\alpha_{n}}{\nu}
$$

In numerical simulations with random initial states usually periodic boundary conditions (pbc) are supposed. The fact that pbc allow only an even number of kinks does not matter except under the conditions of FSS close to the PC point as eventually for all samples the ordering becomes perfect (depletion of kinks) and trivially $\frac{\gamma}{\nu}=\frac{\Theta}{\nu_{\perp}}=1$. Because of the same reason it is not possible to find the $L$-dependence of the kink density, which breaks down as a 
function of time. The proper procedure is to use antiperiodic boundary conditions(apbc), which choice allows only an odd number of kinks (i.e. all samples are surviving) provided the updating procedure is carefully done. As in the course of dynamic FSS one reaches fixed points, which coincide with the limiting values for $t \rightarrow \infty$ of $S$ and $n$ above, we will cite our results in the next section.

As we shall see $\frac{\gamma}{\nu}=1$ is valid also in case of antiperiodic boundary conditions, which value together with eq.(11) gives $Z_{c}=1 / x$. Moreover, we shall arrive at $\frac{\beta_{n}}{\nu_{\perp}}=1 / 2$, which, using eqs. (8) and (9), gives $\frac{\Theta}{\nu_{\perp}}=1$ and leads to $Z=1 / x$. Thus $Z_{c}=Z$, the two critical dynamical exponents coincide, as anticipated. It is worth noting that $Z=1 / x$-type relation between dynamic critical exponent and domain growth exponent has been found earlier for various $1 d$ equilibrium Ising systems [23].

\section{Early-time dynamic Monte-Carlo method}

In case of systems quenched to their critical temperature [24 universality and scaling may appear in a quite early stage of time evolution, far from equilibrium, where $\xi$ is still small. Based on the scaling relation for such early time intervals, a new way for measuring static and dynamic exponents has been proposed [19,20. Now we apply this method to get critical exponents for a nonequilibrium phase transition.

Following 19,20] we shall suppose the following relation to hold for the $\mathrm{k}$-th moment of the magnetisation near the critical point of the $1 \mathrm{D}$ spin system:

$$
M^{(k)}\left(t, p_{T}, L\right)=b^{-\frac{k \beta}{\nu}} M^{(k)}\left(b^{-Z} t, b^{1 / \nu} p_{T}, b^{-1} L\right)
$$

where zero initial magnetisation has been considered and $b$ is a rescaling factor $(b=2$ will be chosen). After generating randomly an initial configuration, the system is let to evolve according to the nonequilibrium kinetic rule of Section II at $p_{T}=0$. ( We could have included $\epsilon$ in eq.(13) as well, but we will restrict ourselves to the case $\epsilon=0, p_{T}=0$, so it is of no importance here). Average is taken over the initial configurations with zero magnetisation. In order to get sufficiently good statistics averaging has to be performed over very many $\left(10^{5}-10^{6}\right)$ independent initial states, as emphasised by Li et al. 19,20] who applied the method to the $2 \mathrm{~d}$ Ising model. To get the dynamical exponent $Z$ with great accuracy, they proposed calculation of the time-dependent Binder cumulant:

$$
U\left(t, p_{T}, L\right)=1-\frac{M^{(4)}}{3\left(M^{(2)}\right)^{2}}
$$

which behaves at $p_{T}=0$ as

$$
U(t, 0, L)=U\left(b^{-Z} t, 0, L / b\right)
$$

Similarly to eq. (13), $n(t, L)$ can be supposed to behave according to the relation:

$$
n(t, L)=b^{-\frac{\beta_{n}}{\nu_{\perp}}} n\left(b^{-Z} t, b^{-1} L\right)
$$

Besides the triviality of the fixed points reached $\left(M^{(2) *}=1, U^{*}=2 / 3\right)$ the pbc case still leads - by proper fitting - to the value of $Z$ and $\beta$. In case of $M^{(2)}$, using eq. (13), collapsing of curves for different values of $L$ has lead to $\beta=0.00(2), Z=1.75(2)$; see Fig.6. In case of $U(t, 0, L)$, which contains only $Z$ as fitting parameter and thus provides it directly, collapsing of curves could be achieved again with $Z=1.75(1)$. Typically $10^{5}-3.10^{5}$ averages have been performed. 


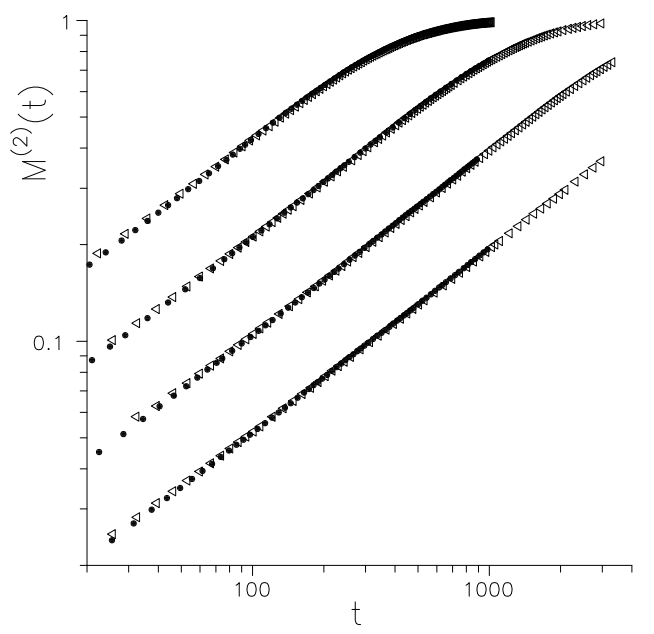

FIG. 6. Collapsing of curves for $M^{(2)}$ with periodic boundary conditions on a double-logarithmic scale. $\triangle$-s are rescaled data while $\bullet$-s are original ones. From top to bottom: L=16( $\triangle)-32(\bullet), 32(\triangle)-64(\bullet), 64(\triangle)-128(\bullet), 128(\triangle)-256(\bullet)$. Number of samples with random initial states in the averaging was typically $10^{5}$

With antiperiodic boundary conditions collapsing of curves has led us to the same results as above concerning the exponents $\beta$ and $Z$. Under these conditions, however, important additional information arises from best fit to time averaged saturation values. Concerning $n(t, L)$, eq.(16), the collapse of curves is illustrated with $L=100,200$ on Fig.7.

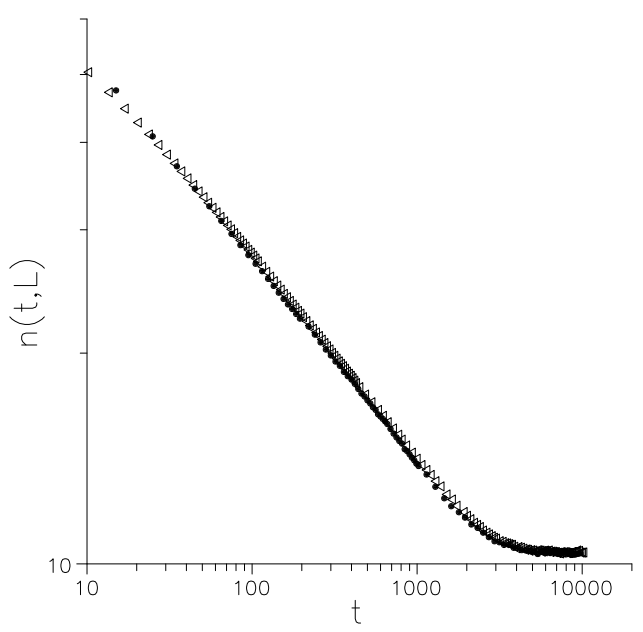

FIG. 7. Collapsing of curves for $n(t, L)$ according to eq.(16). $\triangle$-s: $\mathrm{L}=100$, rescaled; $\bullet-\mathrm{s}: \mathrm{L}=200$. Number of samples in the averaging: $10^{5}$. Boundary condition: antiperiodic, scale: double-logarithmic.

The curves scale together with $Z=1.75(1)$ and $\frac{\beta_{n}}{\nu_{\perp}}=.52(2)$. On the other hand, fitting the time-averaged saturation values for $L=50,64,100,128,200,256$ gives for the kink-density $n(L)=.66 \times L^{-.48(2)}$. Thus we conclude 
$\frac{\beta_{n}}{\nu_{\perp}}\left(=\frac{\alpha_{n}}{\nu}\right)=.50(2)$. This is in accord with the result of Jensen for the $n=4$ BARW [8].

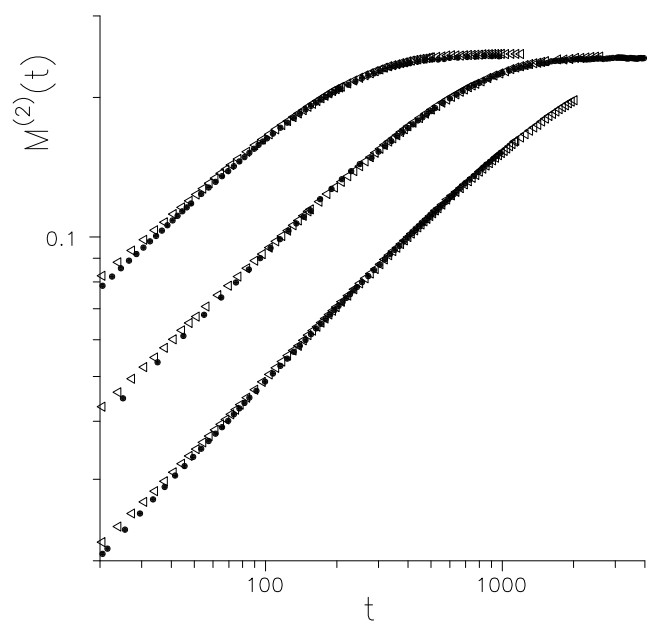

FIG. 8. $M^{(2)}(t, L)$ with apbc. Rescaling with $b=2, \beta=0$ and $Z=1.75$ has resulted in data points marked by $\triangle$-s. Collapsing of curves is shown -from top to bottom- for $\mathrm{L}=32(\triangle)-64(\bullet), \quad 64(\triangle)-128(\bullet), \quad 128(\triangle)-256(\bullet)$. Number of samples in averaging: $10^{5}-3 \times 10^{5}$

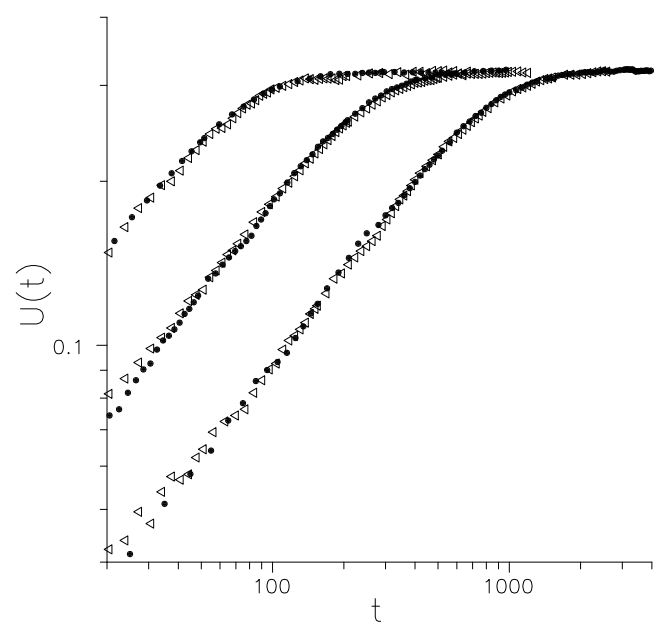

FIG. 9. U(t,L) with apbc for $\mathrm{L}=16(\triangle)-32(\bullet), 32(\triangle)-64(\bullet), 64(\triangle)-128(\bullet)$ (from top to bottom). Best collapse of curves again wit $Z=1.75$. Number of samples in averaging : as for Fig.8.

On Figs.8 and 9., respectively, $M^{(2)}$ and $U$ are seen with antiperiodic boundary conditions for some values of $L$. It is apparent, that $U$ is much more sensitive: here even much more samples in averaging would have been needed to smooth out the curves. The time-averaged saturation values for $L=50,64,100,128,200,256$ lead to $S(L)=.26 L^{.99(1)}$ (Fig.10), thus $\frac{\gamma}{\nu}\left(=\frac{\Theta}{\nu_{\perp}}\right)=.99(1)$. 


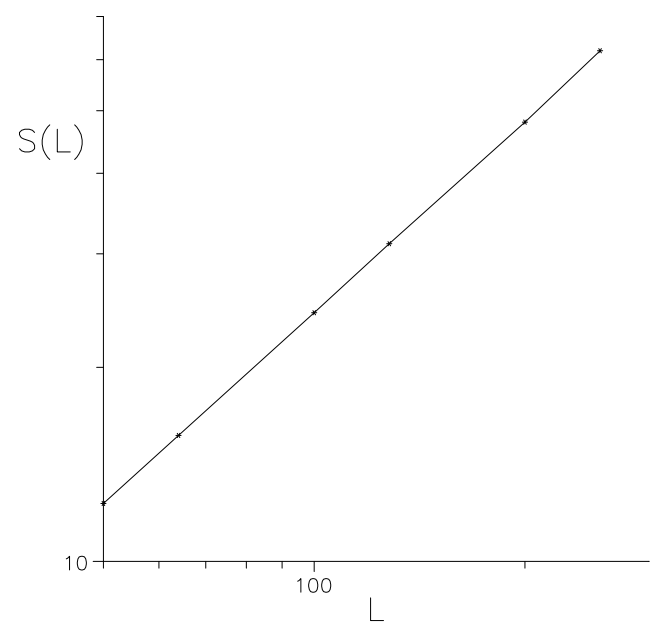

FIG. 10. Time-averaged saturation values $S(L)$ of $M^{(2)}(t, L)$ shown on a double-logarithmic scale. Straight line: power-law fit with $\gamma / \nu=.99$. Averaging has been performed over $10^{5}-3 \times 10^{5}$ independent random initial states with apbc.

The difference as compared with the pbc case shows up in the prefactor ( $.26(1)$ instead of 1.0$)$. For the Binder cumulant $U^{*}=.32(1)$, again differing from the pbc value of $2 / 3$. For the sake of comparison, we have carried out the same kind of simulations for the Ising-Glauber case $\left(p_{e x}=0, \delta=0\right)$ with apbc, and obtained $M_{G l}^{(2) *}=.333(3)$, $n^{*}{ }_{G l}=1.00(5) / L, U^{*}{ }_{G l}=.40(1)$. Here $Z=2$ and $\beta=0$ have given the best fit and similar result can be expected in the whole absorbing phase. It is worth mentioning here, that these fixed-point values can be derived exactly for the Glauber case with the result: $M_{G l}^{(2) *}=1 / 3, n^{*}{ }_{G l}=1 / L, U^{*}{ }_{G l}=.4$ [25].

\section{SYMMETRY-BREAKING FIELD}

It is by now well established that the PC transition has non-DP critical exponents, because of the modulo 2 conservation law. Park and Park [13] have introduced symmetry breaking external field in case of the interacting monomer-dimer model and showed that the DP universality class is recovered if one of the absorbing states is singled out. They also have mentioned of having similar, though - to our knowledge - not yet published data for NEKIM and Grassberger's automata. We have investigated the effect of an external magnetic field $H$ on the NEKIM model with simulations and with the help of the Generalized Mean-Field (GMF) technique as well, both confirming the DP behaviour. The transition probabilities of NEKIM, as given in Sect.II., are modified in the presence of en external magnetic field $\mathrm{H}$ as:

$$
\begin{array}{r}
w_{\text {indif }}^{h}=w_{\text {indif }}\left(1-h s_{i}\right), \\
w_{\text {oppo }}^{h}=w_{\text {oppo }}\left(1-h s_{i}\right), \\
h=\operatorname{th}\left(\frac{H}{k T}\right) .
\end{array}
$$

Here we shall restrict ourselves to $p_{T}=0$. Fig. 11 shows the phase diagram of NEKIM in the $(h, \delta)$ plane, starting at the reference PC point for $h=0$ used in this paper $\left(\delta_{c}=-.395\right)$. 


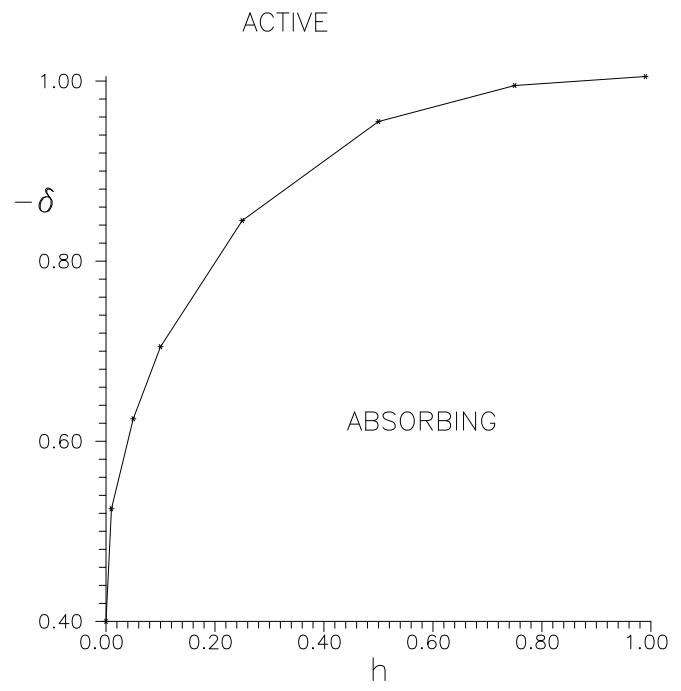

FIG. 11. Phase diagram of NEKIM in the $(h, \delta)$ plane in the presence of an external magnetic field. Parameters of the transition probabilities: $\Gamma=.35, p_{e x}=.3$, see Section II. Naturally, the phase diagram can be drawn symmetrically for negative values of $h$, as well.

We have applied only random initial state simulations to find the points of the line of phase transitions (critical exponents: $\left.\mathrm{y}=.17(2), \beta_{n}=.26(2)\right)$. It is seen, that with increasing field strength the critical point is shifted to more and more negative values of $\delta$.

As to the treatment of the model with the use of the GMF technique, the details of which have been explained in [26 28], in the field-free case we have already obtained estimates for the critical point and the effect of particle exchange earlier [18]. By applying the Coherent Anomaly extrapolation method (CAM) 29] one can extrapolate the critical exponents of the true singular behavior and in [18] we could give a rough estimate for the exponent $\beta_{n}$ of the PC transition based on $N \leq 6$-th order cluster GMF calculation. Now we extend the method for the determination of the exponent of the order-parameter fluctuation as well :

$$
\chi_{n}(\epsilon)=L\left(<n^{2}>-<n>^{2}\right) \sim \epsilon^{-\gamma_{n}} .
$$

The GMF equations have been set up for the steady states of NEKIM in the presence of the H-field. The $N$-block probabilities were determined as the numerical solution of the GMF equations for $N=1, \ldots, 6$. The traditional mean-field solution $(N=1)$ results in stable solutions for the magnetization :

$$
\begin{aligned}
& M=-\frac{h}{\delta}, \quad \text { if } \delta<0 \text { and } h^{2} / \delta^{2}<1 \\
& M=\operatorname{sgn}(h), \quad \text { otherwise. }
\end{aligned}
$$

and for the kink-concentration :

$$
\begin{array}{ll}
n=\frac{1}{2}\left(1-\left(\frac{h}{\delta}\right)^{2}\right), & \text { if } \delta<0 \text { and } h^{2} / \delta^{2}<1 \\
n=0, & \text { otherwise. }
\end{array}
$$

For $N>1$ the solutions can be found numerically only. By increasing the order of approximation the critical point estimates $\delta_{c}(N)$ shift to more negative values similarly to the $H=0$ case. The $\lim _{N \rightarrow \infty} \delta_{c}(h)$ values have been determined with quadratic extrapolation in case of $h=0.01,0.05,0.08,0.1$. The resulting curves for $n(\delta)$ and $\chi_{n}(\delta)$ are shown on Figures 12. and 13., respectively for the case of $h=.1$ in different orders $N$ of the GMF approximation. 


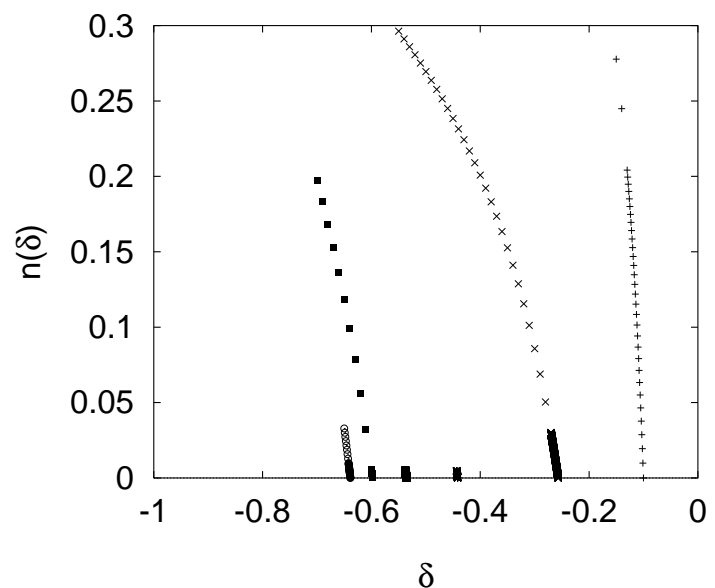

FIG. 12. The kink density in the neighbourhood of the critical point $\delta_{c}(h)$ for $h=.1$. The curves from right to left correspond to $N=1, \ldots, 6$ (level of GMF calculation). The points have been determined with resolution of $10^{-5}$ in $\delta$, in order to be able to extract CAM anomaly coefficients.

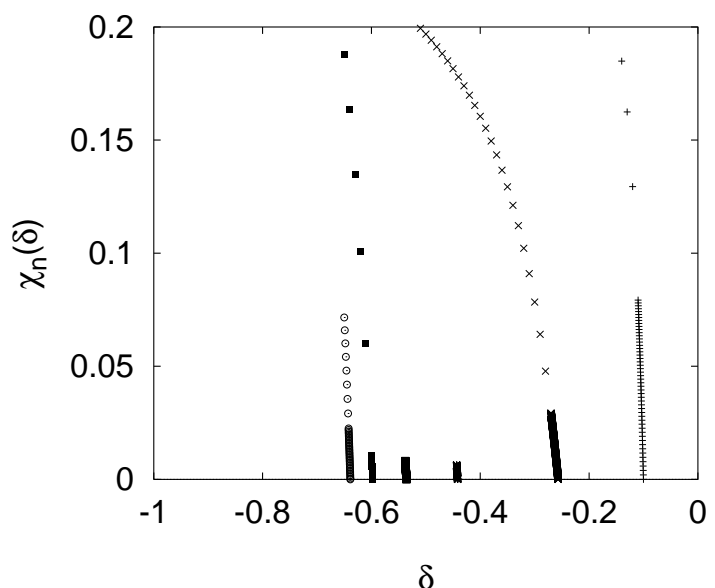

FIG. 13. The same as Fig.12 but for the second moment of the kink density. It is apparent also here that the value of $\delta_{c}(h)$, being equal to $.70(1)$ for $h=.1$ according to simulations, is fairly well approximated by the GMF-results for $N=5,6$.

Naturally, these curves exhibit mean-field type singularity at the critical point :

$$
\begin{gathered}
n(N) \quad \sim \bar{\rho}_{n}\left(\delta / \delta_{c}(N)-1\right)^{\beta_{M F}} \\
\chi_{n}(N) \sim \bar{\chi}_{n}(N)\left(\delta / \delta_{c}(N)-1\right)^{-\gamma_{M F}},
\end{gathered}
$$

with $\beta_{M F}=1$ and $\gamma_{M F}=-1$. According to the CAM (based on scaling) the critical exponents of the true singular behavior can be obtained via the scaling behavior of anomaly factors:

$$
\begin{aligned}
\bar{n}(N) & \sim \Delta^{\beta_{n}-\beta_{M F}} \\
\overline{\chi_{n}}(N) & \sim \Delta^{\gamma_{n}-\gamma_{M F}}
\end{aligned}
$$

where we have used the $\Delta=\left(\delta_{c} / \delta_{c}(N)-\delta_{c}(N) / \delta_{c}\right)$ invariant variable instead of $\epsilon$, that was introduced to make the CAM results independent of using $\delta_{c}$ or $1 / \delta_{c}$ coupling ( [30]). Since the level of the GMF calculation, what we could solve is $N \leq 6$, we have taken into account correction to scaling, and determined the true exponents with non-linear fitting form :

$$
\begin{aligned}
& \bar{n}_{(N)}=a \Delta^{\beta_{n}-\beta_{M F}}+b \Delta^{\beta_{n}-\beta_{M F}+1} \\
& \overline{\chi_{n}}(N)=a \Delta^{\gamma_{n}-\gamma_{M F}}+b \Delta^{\gamma_{n}-\gamma_{M F}+1}
\end{aligned}
$$


where $a$ and $b$ are coefficients to be varied. The results for various $h$-s are shown in table below. For $h=0.0$ we could not determine the $\gamma_{n}$ exponent, because the low level GMF calculations resulted in discontinuous phase transition solutions - what we can not use in the CAM extrapolation - and so we had too few data points to achieve a stable non-linear fitting. Higher order GMF solutions would help, but that requires the solution of a non-linear set of equations with more than 72 independent variables. This problem does not occur for $h \neq 0$; the above results - being based on all $N=1 . .6$ point approximations - are fairly stable.

TABLE I. CAM calculation results

\begin{tabular}{lcccccc}
\hline \hline $\mathrm{h}$ & 0.0 & 0.01 & 0.05 & 0.08 & 0.1 & $\mathrm{DP}$ \\
\hline$\beta_{n}$ & 1.0 & 0.281 & 0.270 & 0.258 & 0.285 & $0.2767(4)$ \\
$\gamma_{n}$ & - & 0.674 & 0.428 & 0.622 & 0.551 & $0.5438(13)$ \\
\hline \hline
\end{tabular}




\section{SUMMARY AND CONCLUSIONS}

Time dependent simulations, FSS and dynamic early-time MC method have been applied here to investigate the behaviour at and in the vicinity of a PC transition of the nonequilibrium kinetic Ising model with the aim to complete earlier results. Emphasis has been put now on the critical properties of the 1d spin system underlying kinks. In this way we have arrived at a more-or-less complete picture of the effect the PC transition exerts on the statics and dynamics of the 1D Ising spin phase transition: we have found $Z=1.75$ (instead of $Z=2.0$ ); $\gamma=\nu=.444$ (instead of $1 / 2$ ). The $\mathrm{PC}$ point is endpoint of a line of first order phase transitions ( by keeping $p_{e x}$ and $\Gamma$ fixed and changing $\delta$ through negative values to $\delta_{c}$ ); , where still $\beta=0$ holds and also Fisher's scaling law: $\gamma=d \nu-2 \beta$ is valid. The PC point has been approached from two directions: from the active phase by changing $\epsilon=\delta_{c}-\delta$ and from the direction of finite temperatures by varying $p_{T}=e^{-4 J / k T}$. The second moment of the magnetization ( structure factor ) provides the 'magnetic' exponents $x, \Theta, \gamma$, which have been found - within error - to be twice as big as the corresponding kink exponents, $y, \beta_{n}, \alpha_{n}$. The cause of this factor of two must lie in the nature of the active phase, of course, and it is sufficient to understand $x=2 y$ (time-dependent exponents at $\mathrm{PC}$ ) as the rest follows from scaling relations.

The idea is to recognize that there are two characteristic growth lengths at PC which have to have the same critical exponents. Namely, the magnetic one $L(t) \propto t^{x}$ (see Introduction) and the cluster size defined through the square-root of $\left\langle R^{2}(t)>\propto t^{z}\right.$. The latter one is obtained by starting either from two neighbouring kink initial states (see e.g. [8]), or from a single kink [7, 9], while the magnetic domains grow in the quenching situation i.e. from random initial states $(T=\infty \rightarrow T=0)$. Both length-exponents are, however connected with $Z$, the dynamic critical exponent, since at PC the only dominant length is the ( time- dependent) correlation length. $\frac{z}{2}=\frac{1}{Z}$ has been shown to follow from scaling in [7] and 31] for one-kink and two-kink initial states, respectively. Presently we have found $x=1 / Z$, thus $x=z / 2$ follows.

Exponents of kink density and cluster growth are connected by a hyperscaling relation first established by Grassberger and de la Torre [1] for the directed percolation transition. In the same form it does not apply to PC transition [8,32], where dependence on the initial state (one or two kinks) manifests itself in two cluster-growth quantities: the kink-number $N(t) \propto t^{\eta}$ and the survival probability $P(t) \propto t^{-\delta}$. (This $\delta$ has, of course, nothing to do with the parameter $\delta$ of NEKIM) In case of odd number of kinks (when $\delta=0$, as all samples survive) hyperscaling reads [7]: $y+\eta=z / 2$ As both $y$ and $\eta$ are exponents of time dependent kink density at PC, they should agree numerically if scaling is valid with a single (time-dependent) characteristic length, and this is indeed the case (see e.g. [8] for simulation data). Consequently $2 y=z / 2=x$ follows from the hyperscaling relation.

For an even number of kinks (two-kink situation) the relation with the random initial-state situation investigated here can be established as follows. The local kink density is defined as $\rho(x, t) \propto t^{\eta-z / 2} f\left(x^{2} / t^{z}\right)$ which divided by $P(t)$ leads to our $n(t)$ of eq.(2) at PC: $n(t) \propto t^{-y}$ with $y=-\eta-\delta+z / 2$. Using the hyperscaling relation valid in the two-kink case, namely $2 \delta+\eta=z / 2, y=\delta$ follows. Thus the hyperscaling relation can be written as $2 y+\eta=z / 2$. Taking into account the result first obtained numerically by Jensen [8] that $\eta=0$ for the PC transition when starting with two kinks, $2 y=z / 2=x$ holds, again.

In this way the factor of two between magnetic and kink exponents found in this paper could be explained as following from scaling.

In the last chapter we have introduced a magnetic field into the NEKIM transition rates and investigated its effect mainly in the framework of generalized mean field approximation. By going up to $\mathrm{N}=6$ order cluster approximation, the expectation that the universality class of the phase transition turns into DP-type [13] has been given support: we have found values for the exponents of the kink density and its second moment which are very close to the corresponding DP values.

\section{ACKNOWLEDGEMENTS}

The authors would like to thank Z.Rácz for numerous helpful remarks and the Hungarian research fund OTKA ( Nos. T017493 and 4012) for support during this study. The simulations were partially carried out on the Fujitsu AP1000 parallel supercomputer.

[1] P.Grassberger, A. de la Torre, Annals of Phys. 122(1979) 373

[2] J.L.Cardy, R.L.Sugar, J.Phys.A13 (1980) L423

[3] H.K.Janssen, Z.Physik B42 (1981) 151

[4] P.Grassberger, Z. Physik B47(1982)365 
[5] H.Takayasu, A.Yu Tretyakov, Phys.Rev.Letts.,68 (1992) 3060

[6] P. Grassberger F.Krause and T. von der Twer J.Phys.A:Math.Gen.17(1984)L105

[7] P. Grassberger, J.Phys. A:Math.Gen. 22 (1989) L1103

[8] Jensen I, Phys.Rev.E50 (1994) 3623

[9] N. Menyhárd, J.Phys.A:Math.Gen.27 (1994) 6139

[10] D.Zhong, D. ben-Avraham, Phys. Letts. A209(1995)333

[11] Kim M.H., Park H.,Critical Behaviour of an Interacting Monomer-Dimer Model, Phys.Rev.Letters 73(1994)2579

[12] H.Park, M.H.Kim, H.Park, Dynamic scaling bahaviour of an interacting monomer-dimer model, Inha University preprint, July 1995

[13] H.Park and H.Park, Critical behaviour of an absorbing phase transition in an interacting monomer-dimer model, Inha University preprint. 1995

[14] Glauber R J J.Math.Phys.4 (1963) 191

[15] see e.g. Kawasaki K: Phase Transitions and Critical Phenomena,Vol.2.,ed.Domb C and Green M S (New York: Academic, 1972) p.443

[16] Droz M., Rácz Z. and Schmidt J., Phys.Rev. A39 (1989) 214

[17] Z.Rácz and Zia K P, Phys.Rev.E 49 (1994) 139

[18] N.Menyhárd and G. Ódor, J.Phys.A:Math.Gen.(1995)

[19] Z.B.Li, L.Schülke, B.Zheng, Phys.Rev.Letters 74 (1995) 3396

[20] Z.B.Li, L.Schülke, B.Zheng, Phys.Rev. E53 (1996)2940

[21] A.Sadiq and K.Binder, Phys.Rev.Letts., 51(1983)674

[22] A.Milchev, K.Binder, D.W.Heermann , Z.PhysB63(1986)521

[23] N.Menyhárd, J.Phys.A:Math.Gen. 23(1990)2147

[24] A.J.Bray, Advances in Physics 43 (1994) 357

[25] Z.Rácz, private communication

[26] Gutowitz H.A., Victor J.D., Knight B.W. ,Physica 28D, (1987), 18

[27] Dickman R., Phys.Rev. A38 (1988) 2588

[28] Szabó G., Szolnoki A. and Bodocs L. Phys.Rev. A 44 (1991) 6375 Szabó G., Ódor G. Phys.Rev. E59 (1994) 2764 and references therein

[29] M. Suzuki, J. Phys. Soc. Jpn. 55, 4205 (1986).

[30] M. Kolesik and M. Suzuki, cond-mat/9411109.

[31] J.F.F.Mendes, R.Dickman, M.Henkel, M.C.Marques, J.Phys.A:Math.Gen.27(1994)3019

[32] I.Jensen, Phys.Rev. A46 (1992) 7393 